\title{
CENTRAL INVARIANTS AND HIGHER INDICATORS FOR SEMISIMPLE QUASI-HOPF ALGEBRAS
}

\author{
SIU-HUNG NG AND PETER SCHAUENBURG
}

\begin{abstract}
In this paper, we define the higher Frobenius-Schur (FS-)indicators for finite-dimensional modules of a semisimple quasi-Hopf algebra $H$ via the categorical counterpart developed in a 2005 preprint. When $H$ is an ordinary Hopf algebra, we show that our definition coincides with that introduced by Kashina, Sommerhäuser, and Zhu. We find a sequence of gauge invariant central elements of $H$ such that the higher FS-indicators of a module $V$ are obtained by applying its character to these elements. As an application, we show that FS-indicators are sufficient to distinguish the four gauge equivalence classes of semisimple quasi-Hopf algebras of dimension eight corresponding to the four fusion categories with certain fusion rules classified by Tambara and Yamagami. Three of these categories correspond to well-known Hopf algebras, and we explicitly construct a quasi-Hopf algebra corresponding to the fourth one using the Kac algebra. We also derive explicit formulae for FS-indicators for some quasi-Hopf algebras associated to group cocycles.
\end{abstract}

\section{INTRODUCTION}

The (degree 2) Frobenius-Schur indicator $\nu_{2}(V)$ of an irreducible representation $V$ of a finite group $G$ has been generalized to simple modules of semisimple Hopf algebras by Linchenko and Montgomery [LM00], to certain $C^{*}$-fusion categories by Fuchs, Ganchev, Szlachányi, and Vecsernyés [FGSV99], and to simple modules of semisimple quasi-Hopf algebras by Mason and the first author [MN05]. A more general version of the Frobenius-Schur Theorem holds for the simple modules of semisimple Hopf algebras or even quasi-Hopf algebras. In particular, the FrobeniusSchur indicator of a simple module is non-zero if, and only if, the simple module is self-dual, and its value can only be 0,1 or -1 .

In proving that \pm 1 are the only possible non-zero values for the Frobenius-Schur indicator of a simple module of a semisimple quasi-Hopf algebra $H$ over $\mathbb{C}$ [MN05], the fact that $H$ - $\mathbf{d}_{\text {fin }}$ is pivotal, proved by Etingof, Nikshych, and Ostrik [ENO], has been used. Based on the pivotal structure, the second author [Sch04] later introduced a categorical definition of degree 2 Frobenius-Schur indicators and gave a different proof of the Frobenius-Schur Theorem for quasi-Hopf algebras.

The higher indicators of irreducible representations of a finite group do not have a direct interpretation as the degree 2 indicators (cf. [Isa94]). The $n$-th FrobeniusSchur indicator of a finite-dimensional module $V$ with character $\chi$ of a semisimple

Received by the editors October 11, 2005.

2000 Mathematics Subject Classification. Primary 16W30, 18D10, 81R05.

The first author was supported by the NSA grant number H98230-05-1-0020.

The second author was supported by a DFG Heisenberg fellowship. 
Hopf algebra $H$ has been defined by Kashina, Sommerhäuser, and Zhu [KSZ] as the value of $\chi$ at the $n$-th Sweedler power of the normalized integral of $H$. It has been shown in their paper that these indicators carry rich information on $H$, as well as its module category. Moreover, the values of the $n$-th indicators are cyclotomic integers in $\mathbb{Q}_{n}$.

In the paper [NS], the authors introduced the notion of higher Frobenius-Schur indicators of an object $V$ in a $\mathbb{C}$-linear pivotal category $\mathcal{C}$. These indicators are invariants of the pivotal category. If $V$ is simple and $\nu_{n}(V) \neq 0$, then the dual object $V^{\vee}$ of $V$ occurs in $V^{\otimes(n-1)}$. Again, the $n$-th indicator of any object in $\mathcal{C}$ is a cyclotomic integer in $\mathbb{Q}_{n}$. In addition, if $\mathcal{C}$ is a pseudo-unitary fusion category over $\mathbb{C}$, then the higher indicators will be preserved by any $\mathbb{C}$-linear monoidal equivalence from $\mathcal{C}$ to any other pseudo-unitary fusion category over $\mathbb{C}$.

In this paper, we continue our study of Frobenius-Schur indicators defined in [NS] for the module category of a semisimple complex quasi-Hopf algebra $H$. It has been proved in [ENO] that $H$-mod fin $_{\text {fin }}$ iseudo-unitary; equivalently, $H$-mod fin $_{\text {fin }}$ admits a unique pivotal structure $j$ for which the pivotal dimension of $V \in H$ - $\mathbf{m o d}_{\text {fin }}$ is the ordinary dimension of $V$. We will refer to this pivotal structure as the canonical pivotal structure on $H$ - $\bmod _{\text {fin }}$. It has been shown in [MN05] that the canonical pivotal structure $j$ is given by the trace-element $g$ of $H$, namely

$$
j_{V}: V \rightarrow V^{\vee \vee}, \quad j_{V}(x)(f)=f\left(g^{-1} x\right) \quad \text { for } x \in V, f \in V^{\vee},
$$

and $g$ can be expressed in terms of the integral, the associator and the antipode of the quasi-Hopf algebra. For the canonical pivotal structure of $H$ - $\mathbf{m o d}_{\text {fin }}$, we prove (Theorem 4.1) that there exists a canonical central element $\mu_{n}(H)$ for each positive integer $n$ such that the $n$-th Frobenius-Schur indicator $\nu_{n}(V)$ of $V$ is given by

$$
\nu_{n}(V)=\chi\left(\mu_{n}(H)\right)
$$

where $\chi$ is the character of the $H$-module $V$. The elements $\mu_{n}(H)$ are invariant under gauge transformations and independent of the choice of antipode of $H$. Moreover, we find an expression for $\mu_{n}(H)$ in terms of the integral, the associator and the antipode of $H$ (Proposition 4.5). These results are extensions of the corresponding degree 2 results in sections 3 and 4 of [MN05]. The formula (0.1) also implies that our definition of higher indicators coincides with the one introduced in [KSZ] when $H$ is a Hopf algebra.

The organization of the paper is as follows: we cover some basic definitions and facts about quasi-Hopf algebras $H$, including some important elements of $H \otimes H$ and identities introduced by Drinfel'd [Dri90], Hausser, and Nill [HN], in Section 1. In Section 2, we prove that two finite-dimensional Hopf algebras over a field $k$ are gauge equivalent if, and only if, their module categories are $k$-linear monoidally equivalent. In addition, if $H$ is semisimple and $k=\mathbb{C}$, then $H$-mod $\min _{\text {fin }}$ is a spherical fusion category with respect to a canonical pivotal structure. Moreover, the canonical pivotal structure of $H$ - mod $_{\text {fin }}$ is preserved by any $\mathbb{C}$-linear monoidal equivalence from $H$ - $\mathbf{m o d}_{\text {fin }}$ to $K$-mod fin for some quasi-Hopf algebra $K$ over $\mathbb{C}$. In Sections 3 and 4 , we define the $(n, r)$-th Frobenius-Schur indicators $\nu_{n, r}(V)$ of a finitedimensional $H$-module $V$. We determine the central elements $\mu_{n}(H)$ whose action on the $H$-modules gives the $n$-th Frobenius-Schur endomorphisms introduced in [NS]. The element $\mu_{n}(H)$ is an invariant under gauge transformations on $H$ and 
$\nu_{n}(V)=\chi\left(\mu_{n}(H)\right)$ where $\chi$ is the character of $V$. As an example, we derive a formula for the higher indicators for the semisimple quasi-Hopf algebra obtained from a semisimple Hopf algebra with a central group-like element of order 2 in Section 5. In Section 6, we use this formula to show that Frobenius-Schur indicators suffice to identify and distinguish the four gauge equivalence classes of non-commutative semisimple quasi-Hopf algebras of dimension 8 whose fusion rules (or $\mathcal{K}(H)$ ) contain an abelian group isomorphic to $\mathbb{Z}_{2} \times \mathbb{Z}_{2}$. The corresponding categories were classified by Tambara and Yamagami. Finally, in Section 7, we obtain formulae for the higher FS-indicators for the dual of the group algebra of a finite group $G$ with quasi-Hopf algebra structure determined by a group 3-cocycle, and for its double, known as the twisted double $D^{\omega}(G)$.

\section{Preliminaries and notation}

In this section, we recall the definition of quasi-Hopf algebras, some properties described in [Dri90] and [Kas95], and some interesting results obtained in [HN], [HN99b], and [HN99a]. In the sequel, we will use the notation introduced in this section.

A quasi-bialgebra over a field $k$ is a quadruple $(H, \Delta, \epsilon, \phi)$, in which $H$ is an algebra over $k, \Delta: H \rightarrow H \otimes H$ and $\epsilon: H \rightarrow k$ are algebra maps, and $\phi \in H^{\otimes 3}$ is the associator. Here our convention of associator $\phi$ is given by the equation

$$
\phi(\Delta \otimes \mathrm{id}) \Delta(h)=(\mathrm{id} \otimes \Delta) \Delta(h) \phi .
$$

A quasi-bialgebra $(H, \Delta, \epsilon, \phi)$ is called a quasi-Hopf algebra if there exist an antialgebra automorphism $S$ of $H$ and elements $\alpha, \beta \in H$ such that for all elements $h \in H$, we have

$$
\begin{gathered}
S\left(h_{(1)}\right) \alpha h_{(2)}=\epsilon(h) \alpha, \quad h_{(1)} \beta S\left(h_{(2)}\right)=\epsilon(h) \beta, \\
\phi^{(1)} \beta S\left(\phi^{(2)}\right) \alpha \phi^{(3)}=1, \quad S\left(\phi^{(-1)}\right) \alpha \phi^{(-2)} \beta S\left(\phi^{(-3)}\right)=1,
\end{gathered}
$$

where $\phi=\phi^{(1)} \otimes \phi^{(2)} \otimes \phi^{(3)}, \phi^{-1}=\phi^{(-1)} \otimes \phi^{(-2)} \otimes \phi^{(-3)}$ and $\Delta(h)=h_{(1)} \otimes h_{(2)}$. In the above equations, the summation notation of the tensors has been suppressed. For simplicity, we will continue to do so in the sequel. The triple $(S, \alpha, \beta)$ or sometimes just the anti-automorphism $S$ is called an antipode for $H$. We will simply write $H$ for a quasi-bialgebra $(H, \Delta, \epsilon, \phi)$ or a quasi-Hopf algebra $(H, \Delta, \epsilon, \phi, \alpha, \beta, S)$.

The module category $H$-mod of the quasi-bialgebra $H$ is a $k$-linear monoidal category, or simply a tensor category. If $H$ is a quasi-Hopf algebra, then the tensor category $H$-mod $_{\text {fin }}$ of all finite-dimensional $H$-modules is rigid, i.e. $H$-mod fin $_{\text {ad- }}$ mits both left and right duality. Given any $V \in H$-mod fin $_{\text {fith }}$ the left $H$-module structure given by $\rho: H \rightarrow \operatorname{End}_{k}(V)$, the left dual $\left(V^{\vee}, \mathrm{ev}, \mathrm{db}\right)$ of $V$ is defined as follows:

(1) $V^{\vee}=\operatorname{Hom}_{k}(V, k)$ with the $H$-action given by $h \mapsto \rho(S(h))^{*}$,

(2) ev: $V^{\vee} \otimes V \rightarrow k$ and $\mathrm{db}: k \rightarrow V \otimes V^{\vee}$ defined by

$$
\mathrm{ev}(f \otimes v)=f(\alpha v) \quad \text { and } \quad \mathrm{db}(1)=\sum_{i} \beta v_{i} \otimes v^{i},
$$

where $\left\{v_{i}\right\}$ is a basis for $V$ and $\left\{v^{i}\right\}$ the corresponding dual basis. 
Similarly, one can define ${ }^{\vee} V=\operatorname{Hom}_{k}(V, k)$ with the left $H$-action given by $h \mapsto$

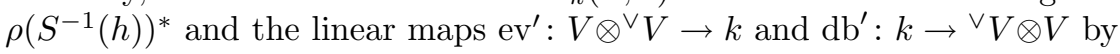

$$
\mathrm{ev}^{\prime}(v \otimes f)=f\left(S^{-1}(\alpha) v\right) \quad \text { and } \quad \mathrm{db}^{\prime}(1)=\sum_{i} v^{i} \otimes S^{-1}(\beta) v_{i}
$$

Then $\left({ }^{\vee} V, \mathrm{ev}^{\prime}, \mathrm{db}^{\prime}\right)$ defines a right dual of $V$ (cf. [Dri90] and [Kas95] for the details).

Following [Kas95], a gauge transformation on a quasi-bialgebra $H=(H, \Delta, \epsilon, \phi)$ is an invertible element $F$ of $H \otimes H$ such that

$$
(\epsilon \otimes \mathrm{id})(F)=1=(\mathrm{id} \otimes \epsilon)(F) .
$$

Using a gauge transformation $F$ on $H$, one can define an algebra map $\Delta^{F}: H \rightarrow$ $H \otimes H$ by

$$
\Delta^{F}(h)=F \Delta(h) F^{-1}
$$

for any $h \in H$, and an invertible element $\phi^{F}$ of $H \otimes H \otimes H$ by

$$
\phi^{F}=(1 \otimes F)(\mathrm{id} \otimes \Delta)(F) \phi(\Delta \otimes \mathrm{id})\left(F^{-1}\right)\left(F^{-1} \otimes 1\right) .
$$

Then $H^{F}=\left(H, \Delta^{F}, \epsilon, \phi^{F}\right)$ is also a quasi-bialgebra. If $H=(H, \Delta, \epsilon, \phi, \alpha, \beta, S)$ is a quasi-Hopf algebra, then so is $H^{F}=\left(H, \Delta^{F}, \epsilon, \phi^{F}, \alpha^{F}, \beta^{F}, S\right)$, where

$$
\alpha^{F}=\sum_{i} S\left(d_{i}\right) \alpha e_{i} \quad \text { and } \quad \beta^{F}=\sum_{i} f_{i} \beta S\left(g_{i}\right)
$$

with $F=\sum_{i} f_{i} \otimes g_{i}$ and $F^{-1}=\sum_{i} d_{i} \otimes e_{i}$.

Two quasi-bialgebras $A$ and $B$ are said to be gauge equivalent if there exists a gauge transformation $F$ on $B$ such that $A$ and $B^{F}$ are isomorphic as quasibialgebras. Let $\sigma: A \rightarrow B^{F}$ be such a quasi-bialgebra isomorphism. Then the functor ${ }_{\sigma}(-): B$-mod $\rightarrow A$-mod, with ${ }_{\sigma} V$ the left $A$-module with the underlying space $V$ and the left $A$-action given by

$$
a \cdot v=\sigma(a) v \quad(a \in A, v \in V),
$$

and ${ }_{\sigma} f=f$ for any map $f$ in $B$-mod, is a $k$-linear equivalence. Let $\xi:{ }_{\sigma} V \otimes{ }_{\sigma} W \rightarrow$ ${ }_{\sigma}(V \otimes W)$ be the linear map

$$
V \otimes W \stackrel{F \cdot}{\longrightarrow} V \otimes W
$$

for any $V, W \in B$-mod. Then $(\sigma(-), \xi$,id $)$ is a $k$-linear monoidal equivalence, or simply a tensor equivalence, from $B$-mod to $A$-mod (cf. [Kas95]).

In [HN], [HN99b] and [HN99a], Frank Hausser and Florian Nill introduced some interesting elements in $H \otimes H$ for a quasi-Hopf algebra $H=(H, \Delta, \epsilon, \phi, \alpha, \beta, S)$ in the course of studying the corresponding theories of quantum double, integral and the fundamental theorem for quasi-Hopf algebras. These elements of $H \otimes H$ are given by

$$
\begin{array}{cl}
q_{R}=\phi^{(1)} \otimes S^{-1}\left(\alpha \phi^{(3)}\right) \phi^{(2)}, & p_{R}=\phi^{(-1)} \otimes \phi^{(-2)} \beta S\left(\phi^{(-3)}\right), \\
q_{L}=S\left(\phi^{(-1)}\right) \alpha \phi^{(-2)} \otimes \phi^{(-3)}, & p_{L}=\phi^{(2)} S^{-1}\left(\phi^{(1)} \beta\right) \otimes \phi^{(3)} .
\end{array}
$$


The elements $q_{L}$ and $p_{L}$ also occurred in [Dri90]. One can show (cf. [HN]) that they obey the relations (for all $h \in H$ )

$$
\begin{aligned}
(h \otimes 1) q_{R} & =\left(1 \otimes S^{-1}\left(h_{(2)}\right)\right) q_{R} \Delta\left(h_{(1)}\right), \\
p_{R}(h \otimes 1) & =\Delta\left(h_{(1)}\right) p_{R}\left(1 \otimes S\left(h_{(2)}\right)\right), \\
(1 \otimes h) q_{L} & =\left(S\left(h_{(1)}\right) \otimes 1\right) q_{L} \Delta\left(h_{(2)}\right), \\
p_{L}(1 \otimes h) & =\Delta\left(h_{(2)}\right) p_{L}\left(S^{-1}\left(h_{(1)}\right) \otimes 1\right),
\end{aligned}
$$

where $\Delta(h)=h_{(1)} \otimes h_{(2)}$. Suppressing the summation symbol and indices again, we write $q_{R}=q_{R}^{(1)} \otimes q_{R}^{(2)}$, etc. These elements also satisfy the identities (cf. [HN]):

$$
\begin{aligned}
& \Delta\left(q_{R}^{(1)}\right) p_{R}\left(1 \otimes S\left(q_{R}^{(2)}\right)\right)=\left(1 \otimes S^{-1}\left(p_{R}^{(2)}\right)\right) q_{R} \Delta\left(p_{R}^{(1)}\right)=1 \otimes 1, \\
& \Delta\left(q_{L}^{(2)}\right) p_{L}\left(S^{-1}\left(q_{L}^{(1)}\right) \otimes 1\right)=\left(S\left(p_{L}^{(1)}\right) \otimes 1\right) q_{L} \Delta\left(p_{L}^{(2)}\right)=1 \otimes 1 .
\end{aligned}
$$

\section{Module CATEgories of QUASI-Hopf Algebras}

In this section, we recall the canonical pivotal structure of the module categories of finite-dimensional semisimple quasi-Hopf algebras over $\mathbb{C}$ and some properties of these tensor categories. We also prove that two finite-dimensional quasi-Hopf algebras are gauge equivalent if, and only if, their module categories are tensor equivalent.

It is well known that if $H$ and $K$ are gauge equivalent quasi-bialgebras, then $H$-mod and $K$-mod are equivalent tensor categories (cf. [Kas95]). The converse for finite-dimensional Hopf algebras was proved in [Sch96]. The quasi-bialgebra case was proved in [EG02, section 6]. Here we give a more straightforward proof for the case of finite-dimensional quasi-Hopf algebras.

Lemma 2.1. Let $H$ be a quasi-Hopf algebra over a field $k, R$ a $k$-algebra and $V$ an $H$-R-bimodule. Then $\theta: H \otimes V \rightarrow H \otimes 。 V$ given by

$$
\theta(h \otimes v)=q_{R}^{(1)} h_{(1)} \otimes S\left(q_{R}^{(2)} h_{(2)}\right) v,
$$

for any $h \in H$ and $v \in V$, is a natural $H$-R-bimodule isomorphism, where ${ }_{\circ} V$ denotes the trivial $H$-module with the underlying space $V$ and the right $R$-actions on $H \otimes V$ and $H \otimes{ }_{\circ} V$ are induced by the right $R$-action on $V$.

Proof. It follows directly from (1.9) that $\theta$ is a natural bimodule homomorphism. Consider the $H$-module map $\bar{\theta}: H \otimes 。 V \rightarrow H \otimes V$ given by

$$
\bar{\theta}(h \otimes v)=h_{(1)} p_{R}^{(1)} \otimes h_{(2)} p_{R}^{(2)} v .
$$

Using (1.9) and (1.11), one can easily verify that $\theta \bar{\theta}=\bar{\theta} \theta=$ id. Hence, the result follows.

Theorem 2.2. Let $H$ be a finite-dimensional quasi-Hopf algebra, and $B$ a quasibialgebra over a field $k$. If $H$-mod and $B$-mod are tensor equivalent (in particular if $B$ is finite-dimensional, and $H$ - $\mathbf{m o d}_{\mathrm{fin}}$ and $B-\mathbf{m o d}_{\mathrm{fin}}$ are tensor equivalent), then $B$ is gauge equivalent to $H$ as quasi-bialgebras.

Proof. Let $\left(\mathcal{F}, \xi, \xi_{0}\right)$ be a tensor equivalence from $H$-mod to $B$-mod. By the Morita Theorems, we may assume that $\mathcal{F}=T \otimes_{H}-$ for some $T \in B$-H-mod such that $T_{H}$ is a progenerator, and in particular, $T$ is finite-dimensional. Moreover, the algebra homomorphism $\sigma^{\prime}: B \rightarrow \operatorname{End}_{H}\left(T_{H}\right)$ given by the $B$-module structure 
of $T$, is an isomorphism. To show that $H \cong B$ as algebras, it suffices to prove that $T \cong H$ as right $H$-modules. By Lemma 2.1 , there exists an $H$ - $H$-bimodule isomorphism $\theta: H \otimes H \rightarrow H \otimes_{\diamond} H$. Thus, we have

$$
T \otimes T \cong \mathcal{F}(H) \otimes \mathcal{F}(H) \stackrel{\xi}{\cong} \mathcal{F}(H \otimes H) \stackrel{\mathcal{F}(\theta)}{\cong} \mathcal{F}\left(H \otimes_{\circ} H\right)=T \otimes_{H} H \otimes_{\circ} H \cong T \otimes_{\circ} H
$$

as left $B$-modules. Obviously, all the above unlabeled isomorphisms are $B-H$ bimodule isomorphisms. Since $\theta$ is an $H$ - $H$-bimodule isomorphism, $\mathcal{F}(\theta)$ is a $B$ $H$-bimodule isomorphism. The naturality of $\xi$ implies that $\xi: \mathcal{F}(H) \otimes \mathcal{F}(H) \rightarrow$ $\mathcal{F}(H \otimes H)$ is also a $B-H \otimes H$-bimodule isomorphism. Thus, we have

$$
T \otimes T \cong T \otimes_{\circ} H
$$

as $B$ - $H$-bimodules, and hence $T \cong H$ as right $H$-modules by the Krull-Schmidt Theorem. Let $\sigma: B \rightarrow H$ be the composition map

$$
B \stackrel{\sigma^{\prime}}{\longrightarrow} \operatorname{End}_{H}\left(T_{H}\right) \cong \operatorname{End}_{H}\left(H_{H}\right) \cong H .
$$

As in (1.6), the algebra map $\sigma$ induces a $k$-linear equivalence $\sigma(-): H$-mod $_{\text {fin }} \rightarrow$ $B$ - mod $_{\text {fin }}$ and $T \cong{ }_{\sigma} H$ as $B$ - $H$-bimodules. The following $B$-module isomorphisms

$$
T \otimes_{H} V \cong{ }_{\sigma} H \otimes_{H} V \cong{ }_{\sigma} V
$$

are natural in $V$ and hence $\sigma(-)$ is $k$-linearly equivalent to $\mathcal{F}$. Therefore, one may assume $\mathcal{F}(-)={ }_{\sigma}(-)$. Note that $\left(\mathcal{F}, z \xi, z^{-1} \xi_{0}\right)$ is also a tensor equivalence for any non-zero scalar $z$. One may further assume $\xi_{0}=\mathrm{id}_{k}$ and so we have $\epsilon_{H} \sigma=\epsilon_{B}$. Let

$$
F^{\prime}=\xi_{H, H}(1 \otimes 1) \quad \text { and } \quad F=\xi_{H, H}^{-1}(1 \otimes 1) .
$$

Since $\xi_{H, H}$ is a $B-H \otimes H$-bimodule isomorphism, we have

$$
\xi_{H, H}(u \otimes v)=\xi_{H, H}(1 \otimes 1)(u \otimes v)=F^{\prime}(u \otimes v)
$$

for all $u, v \in H$, and

$$
F^{\prime}(\sigma \otimes \sigma)\left(\Delta_{B}(b)\right)=\xi(b \cdot(1 \otimes 1))=b \cdot \xi(1 \otimes 1)=\Delta_{H}(\sigma(b)) F^{\prime}
$$

for all $b \in B$. By naturality again, $\xi_{X, Y}=F^{\prime}$. for any free $H$-modules $X$ and $Y$. Moreover, we have

$$
F F^{\prime}=\xi^{-1}(1 \otimes 1)\left(F^{\prime}\right)=\xi^{-1}\left(F^{\prime}\right)=\xi^{-1}(\xi(1 \otimes 1))=1 \otimes 1
$$

and, similarly, $F^{\prime} F=1 \otimes 1$. Therefore, $F$ is invertible in $H \otimes H$. Finally, by the commutativity of the diagrams
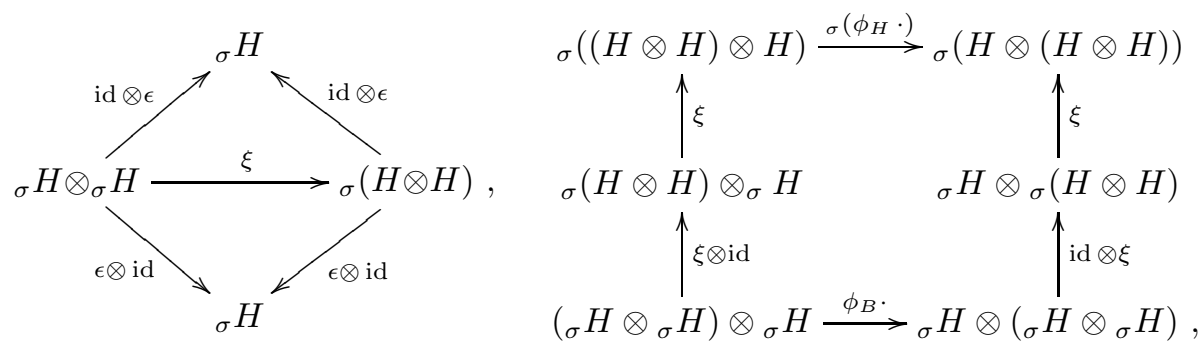

we obtain

$$
1=\left(\mathrm{id} \otimes \epsilon_{H}\right)(F)=\left(\epsilon_{H} \otimes \mathrm{id}\right)(F),
$$

and

$$
(\sigma \otimes \sigma \otimes \sigma)\left(\phi_{B}\right)=(1 \otimes F)(\mathrm{id} \otimes \Delta)(F) \phi_{H}(\Delta \otimes \mathrm{id})\left(F^{-1}\right)\left(F^{-1} \otimes 1\right) .
$$


Therefore, $F$ is a gauge transformation on $H$ and $\sigma: B \rightarrow H^{F}$ is quasi-bialgebra isomorphism.

Recall that a pivotal structure for a rigid monoidal category $\mathcal{C}$ is an isomorphism $j: I d \rightarrow(-)^{\vee \vee}$ of monoidal functors. A pivotal monoidal category is a rigid monoidal category with a pivotal structure.

Assume that $H$ is a finite-dimensional semisimple quasi-Hopf algebra over $\mathbb{C}$. It follows from [ENO, Section 8] that $H$ - $\bmod _{\text {fin }}$ admits a unique pivotal structure such that the left pivotal dimension $\operatorname{ptr}^{\ell}\left(\mathrm{id}_{V}\right)$ of any finite-dimensional $H$-module $V$ is identical to its usual dimension $\operatorname{dim}(V)$, where

$$
\underline{\operatorname{ptr}^{\ell}}(f):=\left(\mathbb{C} \stackrel{\mathrm{db}}{\longrightarrow} V \otimes V^{\vee} \stackrel{j_{V} \otimes \mathrm{id}_{V} \vee}{\longrightarrow} V^{\vee \vee} \otimes V^{\vee} \stackrel{\mathrm{ev}}{\longrightarrow} \mathbb{C}\right)
$$

for any $f \in \operatorname{End}_{H}(V)$. We will refer to the pivotal structure described above as the canonical pivotal structure in the sequel. The canonical pivotal structure will be automatically preserved by any tensor equivalence to any other pseudo-unitary fusion category over $\mathbb{C}$. It can be described explicitly in terms of the trace element $g_{H}$ of $H$ (cf. [MN05]).

Remark 2.3. Let $V \in H$ - $\bmod _{\text {fin }}$ and let $V=\bigoplus_{i} X_{i}$ be a decomposition of $V$ as a direct sum of simple $H$-modules $X_{i}$. Let $\iota_{i}: X_{i} \rightarrow V$ and $\pi_{i}: V \rightarrow X_{i}$ be the embeddings and projections associated with the decomposition. For any $f \in$ $\operatorname{End}_{H}(V)$, we have $\pi_{i} f \iota_{i}=f_{i} \operatorname{id}_{X_{i}}$ for some scalar $f_{i} \in \mathbb{C}$ by Schur's Lemma. Therefore,

$$
\underline{\operatorname{ptr}}^{\ell}(f)=\sum_{i} \underline{\operatorname{ptr}}\left(f \iota_{i} \pi_{i}\right)=\sum_{i} \underline{\operatorname{ptr}}{ }^{\ell}\left(\pi_{i} f \iota_{i}\right)=\sum_{i} f_{i} \underline{\operatorname{ptr}}\left(\operatorname{id}_{X_{i}}\right)=\sum_{i} f_{i} \operatorname{dim}\left(X_{i}\right),
$$

which is identical to the usual trace of $f$. In particular,

$$
\underline{\operatorname{ptr}^{\ell}}(f)=\underline{\operatorname{ptr}^{\ell}}\left(f^{\vee}\right)=\underline{\operatorname{ptr}^{r}}(f) .
$$

Therefore, $H$-mod $\mathbf{m}_{\text {fin }}$ is a (non-strict) spherical fusion category over $\mathbb{C}$. This observation has been proved, in a more general context, by Müger [Müg03, Lemma $2.8]$.

In [ENO, Section 8] the existence of the canonical pivotal structure on $H$-mod fin $_{\text {fin }}$ is deduced from the fact that $H$-mod $\mathbf{m}_{\text {fin }}$ is pseudo-unitary; conversely, it is not hard to see that a spherical fusion category with positive pivotal dimensions is pseudounitary in the sense of [ENO].

Proposition 2.4. Let $H$ and $K$ be gauge equivalent finite-dimensional semisimple quasi-Hopf algebras over $\mathbb{C}$. Then every tensor equivalence from $H$-mod $\bmod _{\text {fin }}$ to $K-\bmod _{\text {fin }}$ preserves their canonical pivotal structures.

Proof. Since $H$ - $\mathbf{m o d}_{\text {fin }}$ and $K$-mod $\mathbf{d}_{\text {fin }}$ are pseudo-unitary, the statement follows immediately from [NS, Corollary 6.2].

\section{Frobenius-SCHUR INDiCATORS FOR SEMISIMPLE QUASI-HopF ALGEBRAS}

In this section, we recall the definition of Frobenius-Schur indicators of an object in a linear pivotal category (cf. [NS] for the details). We then give a definition of higher Frobenius-Schur indicators for any finite-dimensional representations of a semisimple quasi-Hopf algebra $H$ over $\mathbb{C}$ using the canonical pivotal structure of 
$H$-mod $\mathbf{d}_{\text {fin }}$ described in Section 2. It follows from [NS] that these indicators are invariants of the tensor category $H$ - mod $_{\text {fin }}$ and hence gauge invariants of $H$.

Let $\mathcal{C}$ be a finite $k$-linear pivotal category, that is, a $k$-linear rigid monoidal category with a pivotal structure $j: I d \rightarrow(-)^{\vee \vee}$ such that $\mathcal{C}(V, W)$ is a finitedimensional $k$-linear space for all $V, W \in \mathcal{C}$. We denote by $V^{\otimes n}$ the $n$-fold tensor power of an object $V \in \mathcal{C}$ with rightmost parentheses; thus $V^{\otimes 0}=I$, the neutral object of $\mathcal{C}$, and $V^{\otimes(n+1)}=V \otimes V^{\otimes n}$. There is a unique isomorphism

$$
\Phi^{(n)}: V^{\otimes(n-1)} \otimes V \rightarrow V^{\otimes n}
$$

composed of instances of the associativity isomorphisms $\Phi$; explicitly $\Phi^{(1)}$ is the identity, and

$$
\Phi^{(n+1)}=\left(\left(V \otimes V^{\otimes(n-1)}\right) \otimes V \stackrel{\Phi}{\longrightarrow} V \otimes\left(V^{\otimes(n-1)} \otimes V\right) \stackrel{V \otimes \Phi^{(n)}}{\longrightarrow} V^{\otimes(n+1)}\right) .
$$

Define $A: \mathcal{C}(I, V \otimes W) \rightarrow \mathcal{C}\left(V^{\vee}, W\right)$ and $T_{V W}: \mathcal{C}\left(V^{\vee}, W\right) \rightarrow \mathcal{C}\left(W^{\vee}, V\right)$ for $V, W$ $\in \mathcal{C}$ by

$$
\begin{gathered}
A(f)=\left(V^{\vee} \stackrel{V^{\vee} \otimes f}{\longrightarrow} V^{\vee} \otimes(V \otimes W) \stackrel{\Phi^{-1}}{\longrightarrow}\left(V^{\vee} \otimes V\right) \otimes W \stackrel{\mathrm{ev} \otimes W}{\longrightarrow} W\right), \\
T_{V W}(f)=\left(W^{\vee} \stackrel{f^{\vee}}{\longrightarrow} V^{\vee \vee} \stackrel{j_{V}^{-1}}{\longrightarrow} V\right),
\end{gathered}
$$

and put

$$
\begin{aligned}
& E_{V W}=\left(\mathcal{C}(I, V \otimes W) \stackrel{A}{\longrightarrow} \mathcal{C}\left(V^{\vee}, W\right) \stackrel{T_{V W}}{\longrightarrow} \mathcal{C}\left(W^{\vee}, V\right) \stackrel{A^{-1}}{\longrightarrow} \mathcal{C}(I, W \otimes V)\right), \\
& E_{V}^{(n)}=\left(\mathcal{C}\left(I, V^{\otimes n}\right) \stackrel{E_{V, V} \otimes(n-1)}{\longrightarrow} \mathcal{C}\left(I, V^{\otimes(n-1)} \otimes V\right) \stackrel{\mathcal{C}\left(I, \Phi^{(n)}\right)}{\longrightarrow} \mathcal{C}\left(I, V^{\otimes n}\right)\right) .
\end{aligned}
$$

Note that $E_{V}^{(1)}=\operatorname{id}_{\mathcal{C}(I, V)}$ as $I^{\vee \vee}=I$. Following [NS], for any positive integers $n, r$, the $(n, r)$-th Frobenius-Schur indicator of $V$ is the scalar

$$
\nu_{n, r}(V)=\operatorname{Tr}\left(\left(E_{V}^{(n)}\right)^{r}\right) .
$$

We will call $\nu_{n}(V):=\nu_{n, 1}(V)$ the $n$-th Frobenius-Schur indicator of $V$. Now, we can define the Frobenius-Schur indicators for the representations of a semisimple quasi-Hopf algebra over $\mathbb{C}$.

Definition 3.1. Let $H$ be a semisimple quasi-Hopf algebra over $\mathbb{C}$ and let $\mathcal{C}$ be the spherical category $H$-mod $\mathbf{m}_{\text {fin }}$ with the canonical pivotal structure described at the end of Section 2. For any $V \in \mathcal{C}$, we call $\nu_{n, r}(V)$ the $(n, r)$-th Frobenius-Schur indicator of $V$ and call $\nu_{n}(V)$ the $n$-th Frobenius-Schur indicator of $V$.

Proposition 3.2. Let $H, K$ be gauge equivalent finite-dimensional semisimple quasi-Hopf algebras over $\mathbb{C}$ via the gauge transformation $F$ on $H$ and the quasibialgebra isomorphism $\sigma: K \rightarrow H^{F}$. For any tensor equivalence $\mathcal{F}$ from $H$-mod min $_{\text {fin }}$ to $K-\bmod _{\text {fin }}$,

$$
\nu_{n, r}(V)=\nu_{n, r}(\mathcal{F}(V))
$$

for any $V \in H-$ mod $_{\text {fin }}$ and positive integers $n, r$. In particular,

$$
\nu_{n, r}(V)=\nu_{n, r}\left({ }_{\sigma} V\right) .
$$

Proof. The result follows directly from Proposition 2.4 and [NS, Corollary 4.4]. 
Remark 3.3. Proposition 3.2 implies that the $(n, r)$-th Frobenius-Schur indicators are gauge invariants of $H$.

Remark 3.4. Let $H$ be a semisimple Hopf algebra over $\mathbb{C}$. By a well-known result of Larson and Radford [LR88], the antipode of $H$ is an involution, and so the canonical pivotal structure of $H$ - mod $_{\text {fin }}$ is given by the natural isomorphism $j: V \rightarrow V^{\vee \vee}$ of $\mathbb{C}$-linear spaces. If one identifies $\operatorname{Hom}_{H}(\mathbb{C}, V)$ with the invariant space $V^{H}$ for any $V \in H-$ mod $_{\text {fin }}$, then

$$
E_{V}^{(n)}\left(\sum u_{1} \otimes \cdots \otimes u_{n}\right)=\sum u_{2} \otimes \cdots \otimes u_{n} \otimes u_{1}
$$

for any $\sum u_{1} \otimes \cdots \otimes u_{n} \in\left(V^{\otimes n}\right)^{H}$. By [KSZ, Corollary 2.3], $\nu_{n}(V)=\operatorname{Tr}\left(E_{V}^{(n)}\right)$ is identical to the $n$-th indicator of $V$ defined by Kashina, Sommerhäuser and Zhu [KSZ]. Therefore, the definition of higher indicators given in Definition 3.1 is indeed a generalization of the higher indicators for Hopf algebras.

\section{Frobenius-Schur endomorphisms-CEntral GAUGE INVARIANTS}

In [NS], we have defined the $n$-th Frobenius-Schur endomorphism of an object in a semisimple pivotal monoidal category and related it to the Frobenius-Schur indicators. In the category $H$ - mod $_{\text {fin }}$ for a semisimple quasi-Hopf algebra $H$, the Frobenius-Schur endomorphism is given by multiplication with a central element $\mu_{n}(H) \in H$. In this section we will obtain an explicit formula for this element $\mu_{n}(H)$ in terms of the quasi-Hopf algebra structure and the normalized integral of $H$. In the case of an ordinary Hopf algebra, this formula simplifies to the $n$-th Sweedler power of the integral, so that $\chi\left(\mu_{n}(H)\right)$ specializes to the original definition of $\nu_{n}(V)$ in [KSZ].

Let $k$ be a field and $\mathcal{C}$ a finite $k$-linear semisimple pivotal category with pivotal structure $j: I d \rightarrow(-)^{\vee \vee}$ and neutral object $I$ such that

$$
\mathcal{C}(X, X) \cong \mathcal{C}(I, I) \cong k
$$

for all simple objects $X$ of $\mathcal{C}$. By [NS], there exists a unique natural isomorphism $\tau_{V T}: V \otimes T \rightarrow T \otimes V$ for any $I$-isotypical object $T$ and for any $V \in \mathcal{C}$ such that $\tau_{V I}=\mathrm{id}_{V}$. One can define the $n$-th Frobenius-Schur endomorphism of $V$ as the composition

$$
\begin{aligned}
& \underline{\mathrm{FS}}_{V}^{(n)}=(V \stackrel{U}{\longrightarrow} Y \otimes\left(V^{\otimes(n-1)} \otimes V\right) \stackrel{Y \otimes \Phi^{(n)}}{\longrightarrow} Y \otimes V^{\otimes n} \stackrel{Y \otimes \pi}{\longrightarrow} \\
&\left.Y \otimes\left(V^{\otimes n}\right)^{\text {triv }} \stackrel{\tau}{\longrightarrow}\left(V^{\otimes n}\right)^{\text {triv }} \otimes Y \stackrel{\iota \otimes Y}{\longrightarrow} V^{\otimes n} \otimes Y \stackrel{C}{\longrightarrow} V\right),
\end{aligned}
$$

where

$$
\begin{aligned}
& U=\left(V \stackrel{\mathrm{db}^{\prime} \otimes V}{\longrightarrow}\left(Y \otimes V^{\otimes(n-1)}\right) \otimes V \stackrel{\Phi}{\longrightarrow} Y \otimes\left(V^{\otimes(n-1)} \otimes V\right)\right), \\
& C=\left(\left(V \otimes V^{\otimes(n-1)}\right) \otimes Y \stackrel{\Phi}{\longrightarrow} V \otimes\left(V^{\otimes(n-1)} \otimes Y\right) \stackrel{V \otimes \mathrm{ev}^{\prime}}{\longrightarrow} V\right),
\end{aligned}
$$

$\left(\left(V^{\otimes n}\right)^{\text {triv }}, \iota, \pi\right)$ is the $I$-isotypical component of $V^{\otimes n}$, and $\left(Y, \mathrm{ev}^{\prime}, \mathrm{db}^{\prime}\right)$ is a right dual of $V^{\otimes n}$. Moreover, by [NS, Theorem 7.6],

$$
\nu_{n}(V)=\underline{\operatorname{ptr}}\left(\underline{\mathrm{FS}}_{V}^{(n)}\right),
$$


where

$$
\underline{\operatorname{ptr}}^{\ell}(f):=\left(I \stackrel{\mathrm{db}}{\longrightarrow} V \otimes V^{\vee} \stackrel{f \otimes V^{\vee}}{\longrightarrow} V \otimes V^{\vee} \stackrel{j_{V} \otimes V^{\vee}}{\longrightarrow} V^{\vee \vee} \otimes V^{\vee} \stackrel{\mathrm{ev}}{\longrightarrow} I\right)
$$

for any $f \in \mathcal{C}(V, V)$. In the above equation, the identification $\mathcal{C}(I, I)=k$ has been used.

Now, let $\mathcal{C}=H$-mod $\mathbf{m}_{\text {fin }}$ for some finite-dimensional semisimple quasi-Hopf algebra $H=(H, \Delta, \epsilon, \phi, \alpha, \beta, S)$ over $\mathbb{C}$. As described in Section 1 , for any $V \in \mathcal{C}$, $\left({ }^{\vee} V, \mathrm{ev}^{\prime}, \mathrm{db}^{\prime}\right)$ defines a right dual of $V$. Thus the maps $U$ and $C$ in the definition of the Frobenius-Schur endomorphism can be expressed in terms of $q$ and $p$ as follows. Let $\left\{u_{i}\right\}$ be a basis for $V^{\otimes(n-1)}$ and $\left\{u^{i}\right\}$ its dual basis for $\vee\left(V^{\otimes(n-1)}\right)$. For any $x \in V, u \in V^{\otimes n}$ and $f \in \vee^{\vee}\left(V^{\otimes(n-1)}\right)$, we have

$$
U(x)=u^{i} \otimes p_{L}^{(1)} u_{i} \otimes p_{L}^{(2)} x \quad \text { and } \quad C(x \otimes u \otimes f)=q_{R}^{(1)} f\left(q_{R}^{(2)} u\right) x .
$$

By $[\mathrm{HN}], H$ admits a unique normalized two-sided integral $\Lambda$, that is, the two-sided integral of $H$ such that $\epsilon(\Lambda)=1$. Then the trivial isotypical component $V^{\text {triv }}$ of a finite-dimensional $H$-module $V$ is given by $\Lambda V$, and $\pi(x)=\Lambda x$ defines a retraction of the inclusion map $\iota: V^{\text {triv }} \rightarrow V$.

Let us define

$$
\Delta^{(0)}=\epsilon, \quad \Delta^{(1)}=\mathrm{id}_{H}, \quad \Delta^{(2)}=\Delta, \quad \phi_{1}=1_{H}, \quad \phi_{2}=1_{H} \otimes 1_{H},
$$

and recursively

$$
\Delta^{(n+1)}=\left(\operatorname{id}_{H} \otimes \Delta\right) \Delta^{(n)}, \quad \phi_{n+1}=\left(1 \otimes \phi_{n}\right)\left(\phi^{(1)} \otimes \Delta^{(n-1)}\left(\phi^{(2)}\right) \otimes \phi^{(3)}\right)
$$

for any positive integer $n \geq 2$. Then $\Phi^{(n)}: V^{\otimes(n-1)} \otimes V \rightarrow V^{\otimes n}$ is given by the action of $\phi_{n}$ on $V^{\otimes n}$. For any $h \in H$, we will suppress the summation notation and simply write

$$
h_{(1)} \otimes \cdots \otimes h_{(n)}
$$

for $\Delta^{(n)}(h)$. In this notation, for any $V \in \mathcal{C}$, the action of an element $h$ of $H$ on $V^{\otimes n}$ is given by

$$
h \cdot\left(x_{1} \otimes \cdots \otimes x_{n}\right)=h_{(1)} x_{1} \otimes \cdots \otimes h_{(n)} x_{n}
$$

for $x_{1} \otimes \cdots \otimes x_{n} \in V^{\otimes n}$.

Now we can derive a formula for the $n$-th Frobenius-Schur endomorphism $\underline{F S}_{V}^{(n)}$ in $\mathcal{C}$. By (4.13), we obtain

$$
\begin{array}{r}
\underline{\mathrm{FS}}_{V}^{(n)}(x)=\sum_{i_{1}, \cdots, i_{n-1}} q_{R}^{(1)} \Lambda_{(1)} \phi_{n}^{(1)} p_{L,(1)}^{(1)} x_{i_{1}}\left\langle q_{R,(1)}^{(2)} \Lambda_{(2)} \phi_{n}^{(3)} p_{L,(2)}^{(1)} x_{i_{2}}, x^{i_{1}}\right\rangle \\
\cdot\left\langle q_{R,(2)}^{(2)} \Lambda_{(3)} \phi_{n}^{(4)} p_{L,(3)}^{(1)} x_{i_{3}}, x^{i_{2}}\right\rangle \\
\vdots \\
\cdot\left\langle q_{R,(n-2)}^{(2)} \Lambda_{(n-1)} \phi_{n}^{(n-1)} p_{L,(n-1)}^{(1)} x_{i_{n-1}}, x^{\left.i_{n-2}\right\rangle}\right. \\
\cdot\left\langle q_{R,(n-1)}^{(2)} \Lambda_{(n)} \phi_{n}^{(n)} p_{L}^{(2)} x, x^{i_{n-1}}\right\rangle
\end{array}
$$


where $\left\{x_{i}\right\}$ is a basis for $V$ and $\left\{x^{i}\right\}$ is its dual basis for $V^{*}$. Since $\sum_{i} x_{i}\left\langle v, x^{i}\right\rangle=v$ for all $v \in V$, one can simplify $\underline{\mathrm{FS}}_{V}^{(n)}(x)$ as follows:

$$
\begin{aligned}
\underline{\mathrm{FS}}_{V}^{(n)}(x)= & \left(q_{R}^{(1)} \Lambda_{(1)} \phi_{n}^{(1)} p_{L,(1)}^{(1)}\right) \cdot\left(q_{R,(1)}^{(2)} \Lambda_{(2)} \phi_{n}^{(3)} p_{L,(2)}^{(1)}\right) \\
& \cdots\left(q_{R,(n-2)}^{(2)} \Lambda_{(n-1)} \phi_{n}^{(n-1)} p_{L,(n-1)}^{(1)}\right) \cdot\left(q_{R,(n-1)}^{(2)} \Lambda_{(n)} \phi_{n}^{(n)} p_{L}^{(2)}\right) x \\
= & m\left(\left(q_{R}^{(1)} \otimes \Delta^{(n-1)}\left(q_{R}^{(2)}\right)\right) \cdot \Delta^{(n)}(\Lambda) \cdot \phi_{n} \cdot\left(\Delta^{(n-1)}\left(p_{L}^{(1)}\right) \otimes p_{L}^{(2)}\right)\right) x
\end{aligned}
$$

where $m$ is the multiplication on $H$. Let us define

$$
\mu_{n}(H):=m\left(\left(q_{R}^{(1)} \otimes \Delta^{(n-1)}\left(q_{R}^{(2)}\right)\right) \cdot \Delta^{(n)}(\Lambda) \cdot \phi_{n} \cdot\left(\Delta^{(n-1)}\left(p_{L}^{(1)}\right) \otimes p_{L}^{(2)}\right)\right)
$$

for any integer $n \geq 1$. The following theorem shows that $\mu_{n}(H)$ is a central gauge invariant of $H$.

Theorem 4.1. Let $H=(H, \Delta, \epsilon, \phi, \alpha, \beta, S)$ be a finite-dimensional semisimple quasi-Hopf algebra over $\mathbb{C}$ and $n$ a positive integer. The element $\mu_{n}(H)$ is in the center of $H$, and it is invariant under gauge transformations on $H$. Moreover, for any $V \in H$-mod ${ }_{\text {fin }}$,

$$
\nu_{n}(V)=\chi\left(\mu_{n}(H)\right)
$$

where $\chi$ is the character of $V$. In addition, if both $\alpha$ and $\beta$ are invertible elements of $H$, then the element $\mu_{n}(H)$ is given by

$$
\mu_{n}(H)=m\left(\Delta^{(n)}(\Lambda) \phi_{n}\right)(\beta \alpha)^{-1}=(\beta \alpha)^{-1} m\left(\Delta^{(n)}(\Lambda) \phi_{n}\right)
$$

where $\Lambda$ is the normalized two-sided integral of $H$.

Proof. Since $\underline{\mathrm{FS}}_{H}^{(n)}$ is an $H$-module map, the equality (4.16) implies that $\mu_{n}(H)$ must lie in the center of $H$. It follows from Remark 2.3 and (4.14) that

$$
\nu_{n}(V)=\underline{\operatorname{ptr}}{ }^{\ell}\left(\underline{\mathrm{FS}}_{V}^{(n)}\right)=\operatorname{Tr}\left(\underline{\mathrm{FS}}_{V}^{(n)}\right)=\chi\left(\mu_{n}(H)\right)
$$

where $\operatorname{Tr}\left(\underline{\mathrm{FS}}_{V}^{(n)}\right)$ denotes the usual trace of the linear operator $\underline{\mathrm{FS}}_{V}^{(n)}$.

Let $K$ be another semisimple quasi-Hopf algebra over $\mathbb{C}$ such that $K$ is gauge equivalent to $H$ via the gauge transformation $F$ on $H$ and the quasi-bialgebra isomorphism $\sigma: K \rightarrow H^{F}$. Then $(\sigma(-), F \cdot$, id $)$ is a tensor equivalence from $H$-mod $_{\text {fin }}$ to $K-\bmod _{\text {fin }}$. By [NS, Lemma 7.3], the functor $\sigma^{(-)}$preserves Frobenius-Schur endomorphisms. Therefore,

$$
\sigma\left(\mu_{n}(K)\right) x=\mu_{n}(K) \cdot x=\underline{\mathrm{FS}}_{K}^{(n)}(x)=\underline{\mathrm{FS}}_{\sigma H}^{(n)}(x)={ }_{\sigma}\left(\underline{\mathrm{FS}}_{H}^{(n)}\right)(x)=\mu_{n}(H) x
$$

for all $x \in{ }_{\sigma} H$. Therefore, $\sigma\left(\mu_{n}(K)\right)=\mu_{n}(H)$. In particular, $\mu_{n}\left(H^{F}\right)=\mu_{n}(H)$.

By [MN05, Lemma 3.1], we have the equations

$$
\beta q_{X}^{(1)} \Lambda_{(1)} \otimes q_{X}^{(2)} \Lambda_{(2)}=\Delta(\Lambda)=\Lambda_{(1)} p_{Y}^{(1)} \otimes \Lambda_{(2)} p_{Y}^{(2)} \alpha
$$

for any $X, Y \in\{R, L\}$. Following from (4.18) and the equation

$$
\phi_{n}\left(\Delta^{(n-1)} \otimes \mathrm{id}\right) \Delta(\Lambda)=\Delta^{(n)}(\Lambda) \phi_{n},
$$

we have

$$
\left(\beta q_{R}^{(1)} \otimes \Delta^{(n-1)}\left(q_{R}^{(2)}\right)\right) \cdot \Delta^{(n)}(\Lambda) \cdot \phi_{n} \cdot\left(\Delta^{(n-1)}\left(p_{L}^{(1)}\right) \otimes p_{L}^{(2)} \alpha\right)=\Delta^{(n)}(\Lambda) \phi_{n} .
$$


Since $\mu_{n}(H)$ is in the center of $H$,

$$
\beta \alpha \mu_{n}(H)=\mu_{n}(H) \beta \alpha=\beta \mu_{n}(H) \alpha=m\left(\Delta^{(n)}(\Lambda) \phi_{n}\right) .
$$

In addition, if $\beta$ and $\alpha$ are invertible elements of $H$, the last statement follows.

Remark 4.2. Since two semisimple quasi-Hopf algebras $H, H^{\prime}$ with identical quasibialgebra structures but different antipodes are gauge equivalent via the gauge transformation $1 \otimes 1$ and the quasi-bialgebra isomorphism id ${ }_{H}$, Theorem $4.1 \mathrm{implies}$ that $\mu_{n}(H)=\mu_{n}\left(H^{\prime}\right)$. Therefore, $\mu_{n}(H)$ is independent of the choice of antipode of $H$.

Remark 4.3. Let $H$ be a semisimple Hopf algebra over $\mathbb{C}$. Then by Theorem 4.1 we have $\nu_{n}(V)=\chi\left(m\left(\Delta^{(n)}(\Lambda)\right)\right)=\chi\left(\Lambda^{[n]}\right)$ for a finite-dimensional $H$-module $V$ with character $\chi$. This recovers the formula used to introduce the $n$-th Frobenius-Schur indicator of $V$ in $[\mathrm{KSZ}]$.

Equation (4.17) contains the elements $p_{L}$ and $q_{R}$ which we recalled in (1.7) and (1.8). In fact it turns out that any combination of the $p$ 's and $q$ 's will give the same result. We need the following observation for the proof.

Lemma 4.4. Let $n$ be a positive integer and let $t$ be an element of $H^{\otimes(n+1)}$. For any $G \in H^{\otimes n}$,

$$
m((1 \otimes G) t)=m(t(G \otimes 1))
$$

where $m$ is the multiplication on $H$.

Proof. The statement can be easily verified by direct computation.

Proposition 4.5. Let $H=(H, \Delta, \epsilon, \phi, \alpha, \beta, S)$ be a finite-dimensional semisimple quasi-Hopf algebra over $\mathbb{C}$ and $n$ a positive integer. Then

$$
\mu_{n}(H)=m\left(\left(q_{X}^{(1)} \otimes \Delta^{(n-1)}\left(q_{X}^{(2)}\right)\right) \cdot \Delta^{(n)}(\Lambda) \cdot \phi_{n} \cdot\left(\Delta^{(n-1)}\left(p_{Y}^{(1)}\right) \otimes p_{Y}^{(2)}\right)\right)
$$

for any $X, Y \in\{R, L\}$.

Proof. Let

$$
T_{X, Y}=m\left(\left(q_{X}^{(1)} \otimes \Delta^{(n-1)}\left(q_{X}^{(2)}\right)\right) \cdot \Delta^{(n)}(\Lambda) \cdot \phi_{n} \cdot\left(\Delta^{(n-1)}\left(p_{Y}^{(1)}\right) \otimes p_{Y}^{(2)}\right)\right)
$$

for any $X, Y \in\{R, L\}$. By definition, $T_{R, L}=\mu_{n}(H)$. Recall from [MN05] that

$$
\begin{aligned}
& \Delta(\Lambda) p_{Y}(h \otimes 1)=\Delta(\Lambda) p_{Y}(1 \otimes S(h)), \\
& (1 \otimes h) q_{X} \Delta(\Lambda)=(S(h) \otimes 1) q_{X} \Delta(\Lambda)
\end{aligned}
$$


for all $h \in H$. Then we have

$$
\begin{aligned}
T_{L, L} & =m\left(\left(\mathrm{id} \otimes \Delta^{(n-1)}\right)\left(q_{L}\right) \Delta^{(n)}(\Lambda) \phi_{n}\left(\Delta^{(n-1)} \otimes \mathrm{id}\right)\left(p_{L}\right)\right) \\
& \stackrel{(4.19)}{=} m\left(\left(\mathrm{id} \otimes \Delta^{(n-1)}\right)\left(q_{L}\right) \phi_{n}\left(\Delta^{(n-1)} \otimes \mathrm{id}\right)\left(\Delta(\Lambda) p_{L}\right)\right) \\
& =m\left(\left(q_{L}^{(1)} \otimes 1\right) \phi_{n}\left(\Delta^{(n-1)} \otimes \mathrm{id}\right)\left(\Delta(\Lambda) p_{L}\left(q_{L}^{(2)} \otimes 1\right)\right)\right) \quad \text { by Lemma } 4.4 \\
& \stackrel{(4.20)}{=} m\left(\left(q_{L}^{(1)} \otimes 1\right) \phi_{n}\left(\Delta^{(n-1)} \otimes \mathrm{id}\right)\left(\Delta(\Lambda) p_{L}\left(1 \otimes S\left(q_{L}^{(2)}\right)\right)\right)\right) \\
& =q_{L}^{(1)} m\left(\Delta^{(n)}(\Lambda) \phi_{n}\left(\Delta^{(n-1)} \otimes \mathrm{id}\right)\left(p_{L}\right)\right) S\left(q_{L}^{(2)}\right) \\
& \stackrel{(4.18)}{=} q_{L}^{(1)} m\left(\left(\mathrm{id} \otimes \Delta^{(n-1)}\right)\left(\left(\beta q_{R}^{(1)} \otimes q_{R}^{(2)}\right) \Delta(\Lambda)\right) \phi_{n}\left(\Delta^{(n-1)} \otimes \mathrm{id}\right)\left(p_{L}\right)\right) S\left(q_{L}^{(2)}\right) \\
& =q_{L}^{(1)} \beta \mu_{n}(H) S\left(q_{L}^{(2)}\right) \\
& =q_{L}^{(1)} \beta S\left(q_{L}^{(2)}\right) \mu_{n}(H) \quad \text { by Theorem } 4.1 \\
& =\mu_{n}(H) .
\end{aligned}
$$

Thus, we have $T_{X, L}=\mu_{n}(H)$ for $X \in\{L, R\}$. Now, for $Y=L$ or $R$, we have

$$
\begin{aligned}
T_{X, Y} & =m\left(\left(\mathrm{id} \otimes \Delta^{(n-1)}\right)\left(q_{X}\right) \Delta^{(n)}(\Lambda) \phi_{n}\left(\Delta^{(n-1)} \otimes \mathrm{id}\right)\left(p_{Y}\right)\right) \\
& =m\left(\left(\mathrm{id} \otimes \Delta^{(n-1)}\right)\left(q_{X}\right) \Delta^{(n)}(\Lambda) \phi_{n}\left(1 \otimes p_{Y}^{(2)}\right)\left(\Delta^{(n-1)}\left(p_{Y}^{(1)}\right) \otimes 1\right)\right) \\
& =m\left(\left(\mathrm{id} \otimes \Delta^{(n-1)}\right)\left(\left(1 \otimes p_{Y}^{(1)}\right) q_{X}\right) \Delta^{(n)}(\Lambda) \phi_{n}\left(1 \otimes p_{Y}^{(2)}\right)\right) \quad \text { by Lemma } 4.4 \\
& =m\left(\left(\mathrm{id} \otimes \Delta^{(n-1)}\right)\left(\left(1 \otimes p_{Y}^{(1)}\right) q_{X} \Delta(\Lambda)\right) \phi_{n}\left(1 \otimes p_{Y}^{(2)}\right)\right) \\
& \stackrel{(4.20)}{=} m\left(\left(\mathrm{id} \otimes \Delta^{(n-1)}\right)\left(\left(S\left(p_{Y}^{(1)}\right) \otimes 1\right) q_{X} \Delta(\Lambda)\right) \phi_{n}\left(1 \otimes p_{Y}^{(2)}\right)\right) \\
& =S\left(p_{Y}^{(1)}\right) m\left(\left(\mathrm{id} \otimes \Delta^{(n-1)}\right)\left(q_{X} \Delta(\Lambda)\right) \phi_{n}\right) p_{Y}^{(2)} \\
& =S\left(p_{Y}^{(1)}\right) m\left(\left(\mathrm{id} \otimes \Delta^{(n-1)}\right)\left(q_{X}\right) \Delta^{(n)}(\Lambda) \phi_{n}\right) p_{Y}^{(2)} \\
& \stackrel{(4.18)}{=} S\left(p_{Y}^{(1)}\right) m\left(\left(\mathrm{id} \otimes \Delta^{(n-1)}\right)\left(q_{X}\right) \phi_{n}\left(\Delta^{(n-1)} \otimes \mathrm{id}\right)\left(\Delta(\Lambda)\left(p_{L}^{(1)} \otimes p_{L}^{(2)} \alpha\right)\right)\right) p_{Y}^{(2)} \\
& =S\left(p_{Y}^{(1)}\right) \mu_{n}(H) \alpha p_{Y}^{(2)} \\
& =\mu_{n}(H) .
\end{aligned}
$$

This proves the statement.

\section{Hopf Algebras With CENTRAL GROUP-LIKE ElEMENTS}

Let $H$ be a finite-dimensional Hopf algebra over $\mathbb{C}$ and $G(H)$ the group of all group-like elements of $H$. Let $G$ be a subgroup of $G(H)$ which lies in the center of $H, \omega$ a normalized 3-cocycle on $G$ with coefficients in $\mathbb{C}^{\times}$and $j: G \rightarrow \hat{G}$ a group isomorphism. Then $j$ can be extended to a Hopf algebra isomorphism from $\mathbb{C}[G]$ to $\mathbb{C}[\hat{G}]=\mathbb{C}[G]^{*}$. Let

$$
e_{x}=\frac{1}{|G|} \sum_{y \in G} j(y)(x)^{-1} y .
$$


By the orthogonality of characters of finite groups,

$$
j\left(e_{x}\right)(z)=\frac{1}{|G|} \sum_{y \in G} j(y)(x)^{-1} j(y)(z)=\frac{1}{|G|} \sum_{y \in G} j(y)\left(x^{-1} z\right)=\delta_{x, z} .
$$

Therefore, $\left\{j\left(e_{x}\right) \mid x \in G\right\}$ is the dual basis of $G$ for $\mathbb{C}[G]^{*}$. In particular, $\left\{e_{x}\right\}_{x \in G}$ is the complete set of orthogonal primitive idempotents of $\mathbb{C}[G]$, and we have the equalities

$$
S\left(e_{x}\right)=e_{x^{-1}}, \quad \epsilon\left(e_{x}\right)=\delta_{1, x}, \quad \Delta\left(e_{x}\right)=\sum_{y \in G} e_{x y^{-1}} \otimes e_{y}
$$

for all $x \in G$. One can construct a new quasi-Hopf algebra using these data of $H$. The following lemma is a joint observation with Geoffrey Mason.

Lemma 5.1. Let $H$ be a Hopf algebra over $\mathbb{C}$ and $G$ a subgroup of $G(H)$. If $G$ lies in the center of $H$, then for any normalized 3 -cocycle $\omega$ on $G$ with coefficients in $\mathbb{C}^{\times}$ and for any group isomorphism $j: G \rightarrow \hat{G}$, the tuple $H_{(G, \omega, j)}=(H, \Delta, \epsilon, \phi, \alpha, \beta, S)$ is a quasi-Hopf algebra, where $\phi, \alpha$ and $\beta$ are defined by

$$
\phi=\sum_{x, y, z \in G} \omega(x, y, z)^{-1} e_{x} \otimes e_{y} \otimes e_{z}, \quad \alpha=1, \quad \beta=\sum_{x \in G} \omega\left(x, x^{-1}, x\right) e_{x},
$$

and $\Delta, \epsilon$ and $S$ are the comultiplication, counit and antipode of $H$. If $\omega^{\prime}$ is cohomologous to $\omega$, then $H_{(G, \omega, j)}$ and $H_{\left(G, \omega^{\prime}, j\right)}$ are gauge equivalent quasi-Hopf algebras.

Proof. Note that

$$
\phi^{-1}=\sum_{x, y, z \in G} \omega(x, y, z) e_{x} \otimes e_{y} \otimes e_{z} \quad \text { and } \quad \beta^{-1}=\sum_{x \in G} \omega\left(x, x^{-1}, x\right)^{-1} e_{x} .
$$

Using the fact that $\mathbb{C}[G]$ lies in the center of $H$ and (5.21), it is straightforward to verify that $H_{(G, \omega, j)}$ is a quasi-Hopf algebra. Let $\omega^{\prime}=\omega \delta b$ for some normalized 2-cochain $b$ of $G$ with coefficients in $\mathbb{C}^{\times}$where

$$
\delta b(x, y, z)=\frac{b(y, z) b(x, y z)}{b(x y, z) b(x, y)}
$$

for $x, y, z \in G$. We define

$$
F=\sum_{x, y \in G} b(x, y)^{-1} e_{x} \otimes e_{y} .
$$

Since $b$ is a normalized cochain, $F$ is a gauge transformation on $H_{(G, \omega, j)}$. Moreover,

$$
\phi^{F}=\sum_{x, y, z \in G} \omega(x, y, z)^{-1} \delta b^{-1}(x, y, z) e_{x} \otimes e_{y} \otimes e_{z}=\sum_{x, y, z \in G} \omega^{\prime}(x, y, z)^{-1} e_{x} \otimes e_{y} \otimes e_{z} .
$$

Since $F$ lies in the center of $H \otimes H, \Delta^{F}=\Delta$ and so $H_{\left(G, \omega^{\prime}, j\right)}=H_{(G, \omega, j)}^{F}$ as quasibialgebras.

Remark 5.2. $H$ and $H_{(G, \omega, j)}$ are identical as bialgebras, and so their module categories are linearly equivalent. If $H$ is also semisimple, then $H$ and $H_{(G, \omega, j)}$ have the same fusion rules for their irreducible representations. However, in general, $H$ - $\mathbf{m o d}_{\text {fin }}$ and $H_{(G, \omega, j)}$ - $\mathbf{m o d}_{\text {fin }}$ are not equivalent tensor categories if $\omega$ is not a coboundary. We will see examples for this below. 
Let us further assume that $H$ is a semisimple Hopf algebra over $\mathbb{C}$ with a central group-like element $u$ of order 2 and $G$ is the subgroup generated by $u$. Note that there is exactly one group isomorphism $j$ from $G$ to $\hat{G}$ and $H^{3}\left(G, \mathbb{C}^{\times}\right)$is an abelian group of order 2 .

Consider the function $\omega: G \times G \times G \rightarrow \mathbb{C}^{\times}$defined by

$$
\omega(x, y, z)=\left\{\begin{aligned}
-1 & \text { if } x=y=z=u, \\
1 & \text { otherwise }
\end{aligned}\right.
$$

One can easily verify that $\omega$ is a non-trivial 3-cocycle of $G$ and so $\omega$ represents the unique non-trivial cohomology class of $H^{3}\left(G, \mathbb{C}^{\times}\right)$. We will simply write $H_{u}$ for the quasi-Hopf algebra $H_{(G, \omega, j)}$.

Let $\chi$ be the non-trivial character of $G$. Then $j(u)=\chi$ and so

$$
e_{1}=\frac{1}{2}(1+u), \quad e_{u}=\frac{1}{2}(1-u) \quad \text { and } \quad \beta=e_{1}-e_{u}=u .
$$

We will proceed to obtain a formula of $\mu_{n}\left(H_{u}\right)$. Let us denote the $n$-th Sweedler power of an element $x$ of $H$ by $x^{[n]}$. It is easy to see that

$$
x^{[n]}=\left\{\begin{array}{cl}
x & \text { if } n \text { is odd, } \\
\epsilon(x) 1 & \text { if } n \text { is even }
\end{array}\right.
$$

for any $x \in \mathbb{C}[G]$. In particular,

$$
e_{z}^{[n]}=\left\{\begin{array}{cl}
e_{z} & \text { if } n \text { is odd } \\
\delta_{z, 1} 1 & \text { if } n \text { is even }
\end{array}\right.
$$

for any $z \in G$. Thus, for any positive integer $n$, we have

$$
m\left(\phi^{(1)} \otimes \Delta^{(n)}\left(\phi^{(2)}\right) \otimes \phi^{(3)}\right)=\sum_{x, y, z \in G} \omega(x, y, z)^{-1} e_{x} e_{y}^{[n]} e_{z}=u^{n},
$$

where $m$ is the multiplication of $H$. Since $\mathbb{C}[G]$ is commutative, by (4.15), we have

$$
m\left(\phi_{r}\right)=u^{(r-1)(r-2) / 2}
$$

for all $r \geq 1$. Indeed the formula is clearly correct for $r=1,2$, and for $r \geq 2$ we have inductively

$$
\begin{aligned}
m\left(\phi_{r+1}\right) & =m\left(\left(1 \otimes \phi_{r}\right)\left(\phi^{(1)} \otimes \Delta^{(r-1)}\left(\phi^{(2)}\right) \otimes \phi^{(3)}\right)\right) \\
& =m\left(\phi_{r}\right) m\left(\phi^{(1)} \otimes \Delta^{(r-1)}\left(\phi^{(2)}\right) \otimes \phi^{(3)}\right) \\
& =u^{(r-1)(r-2) / 2} u^{r-1}=u^{r(r-1) / 2} .
\end{aligned}
$$

Proposition 5.3. Let $H$ be a finite-dimensional semisimple Hopf algebra over $\mathbb{C}$ with a central group-like element $u$ of order 2 . Suppose that $V$ is a finite-dimensional simple $H$-module with character $\chi$, and that $\bar{V}$ is the $H_{u}$-module associated with $V$. Then the $n$-th Frobenius-Schur indicator of $\bar{V}$ is given by

$$
\nu_{n}(\bar{V})=\nu_{n}(V) \chi\left(u^{(n-3) n / 2}\right) / \chi(1)
$$

for any positive integer $n$, where $\nu_{n}(V)$ is the $n$-th Frobenius-Schur indicator of $V$ considered as an $\mathrm{H}$-module.

Proof. Since $u$ is in the center of $H, \phi_{n}$ is also in the center of $H^{\otimes n}$. Thus, by Theorem 4.1 and (5.24), we have

$$
\begin{aligned}
\mu_{n}\left(H_{u}\right) & =m\left(\Delta^{(n)}(\Lambda) \phi_{n}\right)(\beta \alpha)^{-1}=m\left(\Delta^{(n)}(\Lambda)\right) m\left(\phi_{n}\right) u^{-1} \\
& =\Lambda^{[n]} u^{(n-2)(n-1) / 2} u^{-1}=\Lambda^{[n]} u^{(n-3) n / 2},
\end{aligned}
$$


where $\Lambda$ is the normalized integral of $H$. The second and the third equalities follow from (5.23) and (5.24). By Theorem 4.1,

$$
\begin{aligned}
\nu_{n}(\bar{V}) & =\chi\left(\Lambda^{[n]} u^{(n-3) n / 2)}\right) \\
& =\chi\left(\Lambda^{[n]}\right) \chi\left(u^{(n-3) n / 2}\right) / \chi(1) \\
& =\nu_{n}(V) \chi\left(u^{(n-3) n / 2}\right) / \chi(1) .
\end{aligned}
$$

The second equality follows from the fact that $u$ acts on $V$ as a scalar.

Example 5.4. As the simplest example, consider $H=\mathbb{C}[G]$, where $G$ is the group of order 2 generated by $u$. Let $V$ be the non-trivial 1-dimensional $H$-module. Since $u$ acts on $V$ as the scalar -1 , we have $\nu_{n}(V)=\frac{1+(-1)^{n}}{2}$ for all positive integers $n$. By Proposition 5.3, we have

$$
\nu_{n}(\bar{V})=\frac{1+(-1)^{n}}{2}(-1)^{n(n-3) / 2}=\cos \left(\frac{n \pi}{2}\right) .
$$

\section{EXAMPLES AND APPLICATIONS}

In [TY98], Tambara and Yamagami have classified the fusion categories over $\mathbb{C}$ with five simple objects $\{a, b, c, d, m\}$ and fusion rules:

(1) $a m=m a=b m=m b=c m=m c=d m=m d=m$,

(2) $m m=a+b+c+d$, and

(3) $\{a, b, c, d\}$ forms an abelian group isomorphic to $\mathbb{Z}_{2} \times \mathbb{Z}_{2}$.

They found that there are four inequivalent such fusion categories. Three of these are tensor equivalent to the module categories of the following Hopf algebras: $\mathbb{C}\left[Q_{8}\right]$, $\mathbb{C}\left[D_{8}\right]$ and the 8-dimensional Kac algebra $K$, where $Q_{8}$ and $D_{8}$ are, respectively, the quaternion group and the dihedral group of order 8. Susan Montgomery raised the question whether one can explicitly construct a quasi-Hopf algebra whose module category is tensor equivalent to the fourth fusion category with the above fusion rules. Such a quasi-Hopf algebra is known to exist because the simple objects in the category have positive integer dimensions, and in principle it can be reconstructed from the description of the category in [TY98]. However, this general approach is bound to be tedious and yield a rather unwieldy result. The question remains whether the gauge equivalence class of quasi-Hopf algebras corresponding to the fourth category contains a nice representative which admits a more conceptual or concise description. In this section, we will answer this question by showing that these four fusion categories are tensor equivalent to

$$
\mathbb{C}\left[D_{8}\right]-\mathbf{m o d}_{\text {fin }}, \quad \mathbb{C}\left[Q_{8}\right]-\bmod _{\text {fin }}, \quad K-\bmod _{\text {fin }}, \quad \text { and } \quad K_{u}-\bmod _{\text {fin }},
$$

where $u$ is the unique order 2 central group-like element of $K$. In particular, we show that $\mathbb{C}\left[D_{8}\right]$ is gauge equivalent to the quasi-Hopf algebra $\mathbb{C}\left[Q_{8}\right]_{u}$, and $\mathbb{C}\left[D_{8}\right]_{u}$ is gauge equivalent to $\mathbb{C}\left[Q_{8}\right]$

Let $H=\mathbb{C}\left[Q_{8}\right], \mathbb{C}\left[D_{8}\right]$ or $K$. Then $H$ has a unique central group-like element $u$ of order 2 and it has only one 2 -dimensional irreducible representation $V$. We proceed to compute the Frobenius-Schur indicators of $V$ in $H$ - $\bmod _{\text {fin }}$ and those of $\bar{V}$ in $H_{u}-\bmod _{\text {fin }}$.

The 8-dimensional Kac algebra $K$ is a semisimple Hopf algebra over $\mathbb{C}$ generated by $x, y, z$ as a $\mathbb{C}$-algebra with the relations:

$$
x^{2}=1, \quad y^{2}=1, \quad z^{2}=\frac{1}{2}(1+x+y-x y), \quad x y=y x, \quad x z=z y, \quad y z=z x .
$$


The coalgebra structure is given by

$$
\begin{gathered}
\Delta(x)=x \otimes x, \quad \Delta(y)=y \otimes y, \quad \epsilon(x)=\epsilon(y)=1, \\
\Delta(z)=\frac{1}{2}(1 \otimes 1+x \otimes 1+1 \otimes y-y \otimes x)(z \otimes z), \quad \epsilon(z)=1 .
\end{gathered}
$$

The antipode of $K$ is determined by

$$
S(x)=x, \quad S(y)=y, \quad S(z)=z .
$$

Note that $u=x y$ is the unique central group-like element of order 2 of $K$ (cf. [Mas95] and [TY98] for more details on this Kac algebra). Let $V$ be the degree 2 irreducible representation of $K$ and $\chi$ the character of $V$. We have $\chi(u)=-2$. The higher Frobenius-Schur indicators of $V$ considered as a $K$-module are given by

$$
\chi\left(\Lambda^{[n]}\right)=1,0,0,0,1,0,2
$$

where $n=2, \ldots, 8$ and $\Lambda$ is the normalized integral of $K$ given by

$$
\Lambda=\frac{1}{8}((1+x+y+x y)+(1+x+y+x y) z) .
$$

By Proposition 5.3, the $n$-th Frobenius-Schur indicators $(n=2, \ldots, 8)$ of $V$ considered as a $K_{u}$-module are

$$
-1,0,0,0,-1,0,2 \text {. }
$$

Since $Q_{8}$ and $D_{8}$ have centers of order 2, each of $\mathbb{C}\left[Q_{8}\right]$ and $\mathbb{C}\left[D_{8}\right]$ has a unique central group-like element $u$ of order 2 . Let $H=\mathbb{C}\left[Q_{8}\right]$ or $\mathbb{C}\left[D_{8}\right], V$ the degree 2 irreducible representation of $H$ and $\chi$ the character of $V$. Then $\chi(u)=-2$.

For $D_{8}$, the Frobenius-Schur indicators $\nu_{n}(V)$ of $V(n=2, \ldots, 8)$ are well known to be

$$
1,0,2,0,1,0,2 \text {. }
$$

The Frobenius-Schur indicators $\nu_{n}(V)$ of $V(n=2, \ldots, 8)$ for $Q_{8}$ are

$$
-1,0,2,0,-1,0,2 \text {. }
$$

By Proposition 5.3, we can complete the following table of the Frobenius-Schur indicators for the 2-dimensional irreducible representation $V$ of these quasi-Hopf algebras.

\begin{tabular}{|c|c|c|c|c|c|c|c|}
\hline & $\nu_{2}(V)$ & $\nu_{3}(V)$ & $\nu_{4}(V)$ & $\nu_{5}(V)$ & $\nu_{6}(V)$ & $\nu_{7}(V)$ & $\nu_{8}(V)$ \\
\hline$K$ & 1 & 0 & 0 & 0 & 1 & 0 & 2 \\
\hline$K_{u}$ & -1 & 0 & 0 & 0 & -1 & 0 & 2 \\
\hline $\mathbb{C}\left[D_{8}\right]$ & 1 & 0 & 2 & 0 & 1 & 0 & 2 \\
\hline $\mathbb{C}\left[D_{8}\right]_{u}$ & -1 & 0 & 2 & 0 & -1 & 0 & 2 \\
\hline $\mathbb{C}\left[Q_{8}\right]$ & -1 & 0 & 2 & 0 & -1 & 0 & 2 \\
\hline $\mathbb{C}\left[Q_{8}\right]_{u}$ & 1 & 0 & 2 & 0 & 1 & 0 & 2 \\
\hline
\end{tabular}

As a conclusion from this table and the results of [TY98], we have the following theorem.

Theorem 6.1. The fusion categories

$$
K-\bmod _{\text {fin }}, \quad K_{u}-\mathbf{m o d}_{\text {fin }}, \quad \mathbb{C}\left[D_{8}\right]-\bmod _{\text {fin }}, \quad \mathbb{C}\left[Q_{8}\right]-\bmod _{\text {fin }}
$$

form a complete set of inequivalent fusion categories with the fusion rules given at the beginning of this section. Moreover, the quasi-Hopf algebra $\mathbb{C}\left[D_{8}\right]_{u}$ is gauge equivalent to $\mathbb{C}\left[Q_{8}\right]$, and $\mathbb{C}\left[Q_{8}\right]_{u}$ is gauge equivalent to $\mathbb{C}\left[D_{8}\right]$ 
Proof. It follows from the above table and Proposition 3.2 that the fusion categories

$$
K-\bmod _{\text {fin }}, \quad K_{u}-\mathbf{m o d}_{\text {fin }}, \quad \mathbb{C}\left[D_{8}\right]-\bmod _{\text {fin }}, \quad \mathbb{C}\left[Q_{8}\right]-\mathbf{m o d}_{\text {fin }}
$$

are inequivalent tensor categories. Since there are in total four inequivalent fusion categories with the same fusion rules given at the beginning of this section (cf. [TY98]), the categories $K-\bmod _{\text {fin }}, K_{u}-\mathbf{m o d}_{\text {fin }}, \mathbb{C}\left[D_{8}\right]-\mathbf{m o d}_{\text {fin }}, \mathbb{C}\left[D_{8}\right]_{u}-\mathbf{m o d}_{\text {fin }}$ account for all these fusion categories. Since the Frobenius-Schur indicators of the degree 2 irreducible representation of $\mathbb{C}\left[Q_{8}\right]_{u}$ are the same as that of $\mathbb{C}\left[D_{8}\right]$ and the fusion rules of $\mathbb{C}\left[Q_{8}\right]_{u}-\mathbf{m o d}_{\text {fin }}$ and $\mathbb{C}\left[D_{8}\right]-\mathbf{m o d}_{\text {fin }}$ are the same, the categories $\mathbb{C}\left[D_{8}\right]-\mathbf{m o d}_{\text {fin }}$ and $\mathbb{C}\left[Q_{8}\right]_{u}-\mathbf{m o d}_{\text {fin }}$ are tensor equivalent. By Theorem 2.2, $\mathbb{C}\left[D_{8}\right]$ and $\mathbb{C}\left[Q_{8}\right]_{u}$ are gauge equivalent quasi-Hopf algebras. Similarly, one can show that the quasi-Hopf algebras $\mathbb{C}\left[Q_{8}\right]$ and $\mathbb{C}\left[D_{8}\right]_{u}$ are also gauge equivalent.

\section{Higher INDICATORS FOR QUASI-HopF ALGEBRAS ASSOCIATED TO GROUP COCYCLES}

In this section we will derive explicit Frobenius-Schur (FS)-indicator formulae for two kinds of quasi-Hopf algebras associated to a 3-cocycle on a finite group $G$. In particular, Bantay's formula for the second FS-indicator for a twisted quantum double of a finite group derived in [MN05] is a special case of these formulae (see also [Ban00] and [Ban97]).

Let $G$ be a finite group and $\omega$ a normalized 3-cocycle on $G$ with coefficients in $\mathbb{C}^{\times}$. One can construct a quasi-Hopf algebra $H(G, \omega)=\left(\mathbb{C}[G]^{*}, \Delta, \epsilon, \phi, \alpha, \beta, S\right)$ where multiplication, identity, comultiplication, counit and antipode are the same as the structure maps of the Hopf algebra $\mathbb{C}[G]^{*}$, and $\phi, \alpha$, and $\beta$ are given by

$$
\phi=\sum_{a, b, c \in G} \omega(a, b, c) e(a) \otimes e(b) \otimes e(c), \quad \alpha=1, \quad \text { and } \quad \beta=\sum_{a \in G} \omega\left(a, a^{-1}, a\right)^{-1} e(a),
$$

where $\{e(x) \mid x \in G\}$ is the dual basis of $G$ for $\mathbb{C}[G]^{*}$. The quantum double of $H(G, \omega)$ is called the twisted quantum double $D^{\omega^{-1}}(G)$.

The quasi-Hopf algebras $H(G, \omega)$. The basis $\{e(g) \mid g \in G\}$ for $H(G, \omega)$ is a complete set of primitive idempotents of $H(G, \omega)$, and its dual basis $\left\{\chi_{x} \mid x \in G\right\}$ is the complete set of irreducible characters of $H(G, \omega)$.

Proposition 7.1. Let $G$ be a finite group and $\omega$ a normalized 3-cocycle on $G$ with coefficients in $\mathbb{C}^{\times}$. For any simple $H(G, \omega)$-module $V_{x}$ with character $\chi_{x}$ and positive integer $n$,

$$
\nu_{n}\left(V_{x}\right)=\delta_{x^{n}, 1} \prod_{r=1}^{n-1} \omega\left(x, x^{r}, x\right) .
$$

In particular, if $n$ is not a multiple of the order $o(x)$ of $x$, then $\nu_{n}\left(V_{x}\right)$ vanishes. $\nu_{o(x)}\left(V_{x}\right)$ is a root of unity whose order is the same as that of the cohomology class $\operatorname{res}_{\langle x\rangle}[\omega] \in H^{3}\left(\langle x\rangle, \mathbb{C}^{\times}\right)$, and $\nu_{s \cdot o(x)}\left(V_{x}\right)=\nu_{o(x)}\left(V_{x}\right)^{s}$ for $s \in \mathbb{N}$.

Proof. Clearly, $e(1)$ is the normalized integral of $H(G, \omega)$. For any $x \in G$ and integer $n \geq 1$,

$$
e(x)^{[n]}=m\left(\Delta^{(n)}(e(x))\right)=\sum_{y^{n}=x} e(y),
$$


where $\Delta^{(1)}=\mathrm{id}$ and $\Delta^{(n+1)}=\left(\mathrm{id}^{\otimes n-1} \otimes \Delta\right) \Delta^{(n)}$ for $n \geq 1$, and $m$ denotes the multiplication of $H(G, \omega)$. By the commutativity of $H(G, \omega)$ and Theorem 4.1, we have

$$
\nu_{n}\left(V_{x}\right)=\chi_{x}\left(e(1)^{[n]} \beta^{-1} m\left(\phi_{n}\right)\right)
$$

where

$$
\phi_{1}=1_{H}, \quad \phi_{2}=1 \otimes 1, \quad \phi_{r+1}=\left(1 \otimes \phi_{n}\right)\left(\mathrm{id} \otimes \Delta^{(r-1)} \otimes \mathrm{id}\right)(\phi) .
$$

for any integer $r \geq 2$. Note that

$$
m\left(\left(\mathrm{id} \otimes \Delta^{(r)} \otimes \mathrm{id}\right)(\phi)\right)=\sum_{a, b, c \in G} \omega(a, b, c) e(a) e(b)^{[r]} e(c)=\sum_{y \in G} \omega\left(y, y^{r}, y\right) e(y) .
$$

Hence, by induction,

$$
m\left(\phi_{n}\right)=\sum_{y \in G} \prod_{r=1}^{n-2} \omega\left(y, y^{r}, y\right) e(y)
$$

Thus, we have

$$
\nu_{n}\left(V_{x}\right)=\chi_{x}\left(\sum_{y^{n}=1} e(y) \omega\left(y, y^{-1}, y\right) \prod_{r=1}^{n-2} \omega\left(y, y^{r}, y\right)\right)=\delta_{x^{n}, 1} \prod_{r=1}^{n-1} \omega\left(x, x^{r}, x\right) .
$$

Following the description in [MS89], the class of the 3-cocycle $\omega_{x}$ of the cyclic subgroup $\langle x\rangle$ of order $N$ defined by

$$
\omega_{x}\left(x^{\ell}, x^{m}, x^{n}\right)=\exp \left(\frac{2 \pi i}{N^{2}} \widehat{\ell}(\widehat{m}+\widehat{n}-\widehat{m+n})\right)
$$

generates the group $H^{3}\left(\langle x\rangle, \mathbb{C}^{\times}\right)$, where $\widehat{n}$ denotes the remainder upon the division of $n$ by $N$. Then we have

$$
\omega_{x}\left(x, x^{r}, x\right)=\exp \left(\frac{2 \pi i}{N^{2}}(\widehat{r}+1-\widehat{r+1})\right)= \begin{cases}\exp \left(\frac{2 \pi i}{N}\right)=\zeta & \text { if } r \equiv-1 \bmod N \\ 1 & \text { otherwise }\end{cases}
$$

Obviously, there is a 3-coboundary $f$ of $G$ such that

$$
\omega f=\omega_{x}^{t} \quad \text { on }\langle x\rangle \text { for some integer } t .
$$

Since $\nu_{n}\left(V_{x}\right)$ is a gauge invariant,

$$
\nu_{N s}\left(V_{x}\right)=\prod_{r=1}^{N s-1} \omega_{x}^{t}\left(x, x^{r}, x\right)=\left(\zeta^{t}\right)^{s} .
$$

Of course, the order of $\zeta^{t}$ is the same as the order of $\operatorname{res}_{\langle x\rangle}[\omega]$.

The twisted quantum double $D^{\omega}(G)$ of a finite group. The twisted quantum double $D^{\omega}(G)$ of $G$ with respect to $\omega$ is the semisimple quasi-Hopf algebra with underlying vector space $\mathbb{C}[G]^{*} \otimes \mathbb{C}[G]$ in which multiplication, comultiplication $\Delta$, 
associator $\phi$, counit $\epsilon$, antipode $S, \alpha$ and $\beta$ are given by

$$
\begin{gathered}
(e(g) \otimes x)(e(h) \otimes y)=\theta_{g}(x, y) \delta_{g^{x}, h} e(g) \otimes x y, \\
\Delta(e(g) \otimes x)=\sum_{h k=g} \gamma_{x}(h, k) e(h) \otimes x \otimes e(k) \otimes x, \\
\phi=\sum_{g, h, k \in G} \omega(g, h, k)^{-1} e(g) \otimes 1 \otimes e(h) \otimes 1 \otimes e(k) \otimes 1, \\
\epsilon(e(g) \otimes x)=\delta_{g, 1}, \quad \alpha=1, \quad \beta=\sum_{g \in G} \omega\left(g, g^{-1}, g\right) e(g) \otimes 1, \\
S(e(g) \otimes x)=\theta_{g^{-1}}\left(x, x^{-1}\right)^{-1} \gamma_{x}\left(g, g^{-1}\right)^{-1} e\left(x^{-1} g^{-1} x\right) \otimes x^{-1},
\end{gathered}
$$

where $\delta_{g, 1}$ is the Kronecker delta, $g^{x}=x^{-1} g x$, and

$$
\begin{aligned}
& \theta_{g}(x, y)=\frac{\omega(g, x, y) \omega\left(x, y,(x y)^{-1} g x y\right)}{\omega\left(x, x^{-1} g x, y\right)}, \\
& \gamma_{g}(x, y)=\frac{\omega(x, y, g) \omega\left(g, g^{-1} x g, g^{-1} y g\right)}{\omega\left(x, g, g^{-1} y g\right)}
\end{aligned}
$$

for any $x, y, g \in G$ (cf. [DPR90]).

By induction, one can show that

$$
\phi_{n}=\sum_{a_{1}, \cdots, a_{n} \in G}\left(\prod_{i=1}^{n-2} \omega\left(a_{i}, a_{i+1} \cdots a_{n-1}, a_{n}\right)^{-1}\right) e\left(a_{1}\right) \otimes 1 \otimes \cdots \otimes e\left(a_{n}\right) \otimes 1
$$

for any integer $n \geq 2$. The normalized integral of $D^{\omega}(G)$ is given by

$$
\Lambda=\frac{1}{|G|} \sum_{x \in G} e(1) \otimes x
$$

Therefore,

$$
\Delta(\Lambda)=\frac{1}{|G|} \sum_{x, a \in G} \gamma_{x}\left(a^{-1}, a\right) e\left(a^{-1}\right) \otimes x \otimes e(a) \otimes x
$$

and, by induction, we have

$$
\Delta^{(n)}(\Lambda)=\frac{1}{|G|} \sum_{\substack{x, a_{1}, \ldots, a_{n} \in G \\ 1=a_{1} \cdots a_{n}}}\left(\prod_{i=1}^{n-1} \gamma_{x}\left(a_{i}, a_{i+1} \cdots a_{n}\right)\right) e\left(a_{1}\right) \otimes x \cdots \otimes e\left(a_{n}\right) \otimes x
$$

for any integer $n \geq 1$. Thus, we have

Proposition 7.2. Let $G$ be a finite group and $\omega$ a normalized 3-cocycle of $G$ with coefficients in $\mathbb{C}^{\times}$. Then, for any integer $n \geq 2$, the $n$-th Frobenius-Schur indicator of any representation $V$ of $D^{\omega}(G)$ with character $\chi$ is given by

$$
\begin{array}{r}
\nu_{n}(V)=\frac{1}{|G|} \sum_{\substack{x, a \in G \\
x-n=(a x-1)^{n}}}\left(\prod_{i=1}^{n-2} \frac{\gamma_{x}\left(a^{x^{i}}, a^{x^{i+1}} \cdots a^{x^{n-1}}\right) \theta_{a}\left(x^{i}, x\right)}{\omega\left(a^{x^{i}}, a^{x^{i+1}} \cdots a^{x^{n-1}}, a^{x^{n}}\right)}\right) \\
\cdot \frac{\gamma_{x}\left(a, a^{-1}\right) \theta_{a}\left(x^{n-1}, x\right)}{\omega\left(a, a^{-1}, a\right)} \cdot \chi\left(e(a) \otimes x^{n}\right) .
\end{array}
$$


Proof. Since both $\alpha$ and $\beta$ of $D^{\omega}(G)$ are invertible, by Theorem 4.1, we have

$$
\mu_{n}\left(D^{\omega}(G)\right)=\beta^{-1} m\left(\Delta^{(n)}(\Lambda) \phi_{n}\right) .
$$

By (7.30) and (7.31), we obtain that

$$
\begin{gathered}
\Delta^{(n)}(\Lambda) \phi_{n}=\frac{1}{|G|} \sum_{\substack{x, a_{1}, \cdots, a_{n} \in G \\
1=a_{1} \cdots a_{n}}}\left(\prod_{i=1}^{n-2} \frac{\gamma_{x}\left(a_{i+1}, a_{i+2} \cdots a_{n}\right)}{\omega\left(a_{i}^{x}, a_{i+1}^{x} \cdots a_{n-1}^{x}, a_{n}^{x}\right)}\right) \cdot \gamma_{x}\left(a_{1}, a_{1}^{-1}\right) \\
\cdot e\left(a_{1}\right) \otimes x \otimes \cdots \otimes e\left(a_{n}\right) \otimes x .
\end{gathered}
$$

Thus we have

$$
\begin{array}{r}
\beta^{-1} m\left(\Delta^{(n)}(\Lambda) \phi_{n}\right)=\frac{1}{|G|} \sum_{\substack{x, a \in G \\
a a^{x} \cdot a^{x^{n-1}=1}}}\left(\prod_{i=1}^{n-2} \frac{\gamma_{x}\left(a^{x^{i}}, a^{x^{i+1}} \cdots a^{x^{n-1}}\right) \theta_{a}\left(x^{i}, x\right)}{\omega\left(a^{x^{i}}, a^{x^{i+1}} \cdots a^{x^{n-1}}, a^{x^{n}}\right)}\right) \\
\cdot \gamma_{x}\left(a, a^{-1}\right) \theta_{a}\left(x^{n-1}, x\right) \cdot \beta^{-1} \cdot\left(e(a) \otimes x^{n}\right) \\
=\frac{1}{|G|} \sum_{\substack{x, a \in G \\
x^{-n}=\left(a x^{-1}\right)^{n}}}\left(\prod_{i=1}^{n-2} \frac{\gamma_{x}\left(a^{x^{i}}, a^{x^{i+1}} \cdots a^{x^{n-1}}\right) \theta_{a}\left(x^{i}, x\right)}{\omega\left(a^{x^{i}}, a^{x^{i+1}} \cdots a^{x^{n-1}}, a^{x^{n}}\right)}\right) \\
\cdot \frac{\gamma_{x}\left(a, a^{-1}\right) \theta_{a}\left(x^{n-1}, x\right)}{\omega\left(a, a^{-1}, a\right)} \cdot\left(e(a) \otimes x^{n}\right) .
\end{array}
$$

The statement follows easily from (7.33) and Theorem 4.1.

Remark 7.3. The formula for the higher indicators may look different if one uses another form of $\mu_{n}(H)$. For example, $\mu_{n}\left(D^{\omega}(G)\right)=\beta^{-1} m\left(\phi_{n}\left(\Delta^{(n-1)} \otimes \mathrm{id}\right) \Delta(\Lambda)\right)$ since $\phi_{n}\left(\Delta^{(n-1)} \otimes \mathrm{id}\right) \Delta(\Lambda)=\Delta^{(n)}(\Lambda) \phi_{n}$. Using this form of the cental invariant $\mu_{n}\left(D^{\omega}(G)\right)$, one will obtain

$$
\begin{aligned}
\nu_{n}(V)=\frac{1}{|G|} \sum_{\left(a x^{-1}\right)^{n}=x^{-n}} & \left(\prod_{i=1}^{n-2} \frac{\gamma_{x}\left(a^{x^{i-1}}, a^{x^{i}} \cdots a^{x^{n-2}}\right) \theta_{a}\left(x^{i}, x\right)}{\omega\left(a^{x^{i-1}}, a^{x^{i}} \cdots a^{x^{n-2}}, a^{x^{n-1}}\right)}\right) \\
& \cdot \frac{\gamma_{x}\left(a^{-x^{n-1}}, a^{x^{n-1}}\right) \theta_{a}\left(x^{n-1}, x\right)}{\omega\left(a, a^{-1}, a\right)} \cdot \chi\left(e(a) \otimes x^{n}\right) .
\end{aligned}
$$

\section{ACKNOWLEDGEMENTS}

The authors are indebted to Susan Montgomery for her interest in their paper and extended correspondence on the results in Sections 5 and 6 . They also thank Geoffrey Mason for his permission to include part of his observation (Lemma 5.1). The first author would like to thank Penn State University and IHÉS for their hospitality during the preparation of this manuscript. The second author thanks the DFG for support by a Heisenberg fellowship. He also thanks the Institute of Mathematics of the University of Tsukuba and Akira Masuoka for their hospitality.

\section{REFERENCES}

[Ban97] Peter Bantay, The Frobenius-Schur indicator in conformal field theory, Phys. Lett. B 394 (1997), no. 1-2, 87-88. MR1436801 (98c:81195)

[Ban00] Frobenius-Schur indicators, the Klein-bottle amplitude, and the principle of orbifold covariance, Phys. Lett. B 488 (2000), no. 2, 207-210. MR1782167 (2001e:81094) 
[DPR90] R. Dijkgraaf, V. Pasquier, and P. Roche, Quasi Hopf algebras, group cohomology and orbifold models, Nuclear Phys. B Proc. Suppl. 18B (1990), 60-72 (1991), Recent advances in field theory (Annecy-le-Vieux, 1990). MR1128130 (92m:81238)

[Dri90] V. G. Drinfel'd, Quasi-Hopf algebras, Leningrad Math. J. 1 (1990), 1419-1457. MR1047964 (91b:17016)

[EG02] Pavel Etingof and Shlomo Gelaki, On families of triangular Hopf algebras, Int. Math. Res. Not. (2002), no. 14, 757-768. MR1891233 (2002m:16036)

[ENO] Pavel Etingof, Dmitri Nikshych, and Viktor Ostrik, On fusion categories, Ann. of Math. (2) 162 (2005), no. 2, 581-642. MR2183279 (2006m:16051)

[FGSV99] J. Fuchs, A. Ch. Ganchev, K. Szlachányi, and P. Vecsernyés, $S_{4}$ symmetry of $6 j$ symbols and Frobenius-Schur indicators in rigid monoidal $C^{*}$ categories, J. Math. Phys. 40 (1999), no. 1, 408-426. MR1657800 (99k:81111)

[HN] Frank Hausser and Florian Nill, Integral theory for quasi-Hopf algebras, preprint arXiv.math.QA/9904164, 1999.

[HN99a] Diagonal crossed products by duals of quasi-quantum groups, Rev. Math. Phys. 11 (1999), no. 5, 553-629. MR1696105 (2000d:81069)

[HN99b] Doubles of quasi-quantum groups, Comm. Math. Phys. 199 (1999), no. 3, 547-589. MR1669685 (2000a:16075)

[Isa94] I. Martin Isaacs, Character theory of finite groups, Dover Publications Inc., New York, 1994, Corrected reprint of the 1976 original [Academic Press, New York; MR0460423 (57:417)]. MR1280461

[Kas95] Christian Kassel, Quantum groups, Springer-Verlag, New York, 1995. MR1321145 (96e:17041)

[KSZ] Yevgenia Kashina, Yorck Sommerhäuser, and Yongchang Zhu, On higher FrobeniusSchur indicators, Mem. Amer. Math. Soc. 181 (2006), no. 855, viii+65. MR2213320

[LM00] V. Linchenko and S. Montgomery, A Frobenius-Schur theorem for Hopf algebras, Algebr. Represent. Theory 3 (2000), no. 4, 347-355, Special issue dedicated to Klaus Roggenkamp on the occasion of his 60th birthday. MR1808131 (2001k:16073)

[LR88] Richard G. Larson and David E. Radford, Finite-dimensional cosemisimple Hopf algebras in characteristic 0 are semisimple, J. Algebra 117 (1988), no. 2, 267-289. MR957441 (89k:16016)

[Mas95] Akira Masuoka, Semisimple Hopf algebras of dimension 6, 8, Israel J. Math. 92 (1995), no. 1-3, 361-373. MR1357764 (96j:16045)

[MN05] Geoffrey Mason and Siu-Hung Ng, Central invariants and Frobenius-Schur indicators for semisimple quasi-Hopf algebras, Adv. Math. 190 (2005), no. 1, 161-195. MR2104908 (2005h:16066)

[MS89] Gregory Moore and Nathan Seiberg, Classical and quantum conformal field theory, Comm. Math. Phys. 123 (1989), no. 2, 177-254. MR1002038 (90e:81216)

[Müg03] Michael Müger, From subfactors to categories and topology. I. Frobenius algebras in and Morita equivalence of tensor categories, J. Pure Appl. Algebra 180 (2003), no. 1-2, 81-157. MR1966524 (2004f:18013)

[NS] Siu-Hung Ng and Peter Schauenburg, Higher Frobenius-Schur indicators for pivotal categories, Contemp. Math., Amer. Math. Soc., in press.

[Sch96] Peter Schauenburg, Hopf bi-Galois extensions, Comm. Algebra 24 (1996), no. 12, 37973825. MR1408508 (97f:16064)

[Sch04] On the Frobenius-Schur indicators for quasi-Hopf algebras, J. Algebra 282 (2004), no. 1, 129-139. MR2095575 (2005h:16068)

[TY98] Daisuke Tambara and Shigeru Yamagami, Tensor categories with fusion rules of selfduality for finite abelian groups, J. Algebra 209 (1998), no. 2, 692-707. MR1659954 (2000b:18013)

Department of Mathematics, Iowa State University, Ames, Iowa 50011

E-mail address: rng@iastate.edu

Mathematisches Institut der Universität München, Theresienstr. 39, 80333 München, GERMANY

E-mail address: schauenburg@math.lmu.de 